\title{
Polysèmes
}

Revue d'études intertextuelles et intermédiales

\section{Le passage des lettres aux poèmes chez Keats ou comment transposer l'émotion amoureuse}

\section{Oriane Monthéard}

\section{(2) OpenEdition}

\section{Journals}

Édition électronique

URL : http://journals.openedition.org/polysemes/672

DOI : $10.4000 /$ polysemes. 672

ISSN : 2496-4212

Éditeur

SAIT

\section{Édition imprimée}

Date de publication : 1 janvier 2012

Pagination : 103-127

ISSN : 0999-4203

\section{Référence électronique}

Oriane Monthéard, "Le passage des lettres aux poèmes chez Keats ou comment transposer l'émotion amoureuse », Polysèmes [En ligne], 12 | 2012, mis en ligne le 01 mars 2015, consulté le 20 avril 2019. URL : http://journals.openedition.org/polysemes/672 ; DOI : 10.4000/polysemes.672

Ce document a été généré automatiquement le 20 avril 2019

Polysèmes 


\title{
Le passage des lettres aux poèmes chez Keats ou comment transposer l'émotion amoureuse
}

\author{
Oriane Monthéard
}

1 La correspondance de Keats ${ }^{1}$ est un texte frontière non dénué de littérarité car il engage celui qui écrit dans un rapport à l'écriture dynamisée par des intentions esthétiques plus ou moins avouées et plus ou moins abouties. Si la motivation d'origine n'est autre que de communiquer avec les correspondants, elle devient inséparable d'un désir de bien écrire. Ainsi, quand Keats s'adresse à son destinataire, il lui importe le plus souvent de le convaincre, de le distraire, de le séduire, voire de susciter en lui une émotion forte. Ces diverses fonctions ressemblent fort au résultat que tout artiste cherche à atteindre. Donc, si la correspondance de Keats ne peut se voir attribuer le statut d'œuvre au sens strict, il est non seulement possible mais souhaitable d'observer les lettres d'abord indépendamment de l'œuvre pour leur rhétorique, leur structure et leur style.

Dans sa correspondance, espace de liberté à l'abri du regard du public, il est naturel que Keats se livre et offre à son destinataire ses états d'âme, autrement dit ses émotions. Les lettres sont ainsi au plus près de l'émotion, de l'intimité et de sa vie intérieure mais elles s'en distinguent car elles en sont une première reconstitution. Surtout, étudier l'émotion permet de mettre au jour un des aspects les plus essentiels de ces lettres. En effet, quelle que soit sa nature, cette émotion ouvertement énoncée se reconnaît à des modifications dans une écriture épistolaire souvent consacrée au récit des allées et venues, au compte rendu des visites, à des anecdotes, ou à des réflexions sur des sujets divers. Lorsque dans une lettre surgit une émotion, elle en modifie le ton, la texture, la diction, le style. Par exemple, l'épistolier s'emballe et sa lettre se déstructure, ou au contraire il tente d'organiser son propos afin de minimiser l'ampleur du sentiment qui l'envahit. Le rapport de l'épistolier à son écriture se modifie, car elle devient le mode d'expression d'une expérience intime complexe dont la mise en mots ne va pas de soi. Ce travail sur l'écriture renforce alors le degré de littérarité de la correspondance. 
Ces émotions, et notamment le sentiment amoureux sur lequel je vais me concentrer, prennent une place très particulière car elles sont présentes également dans la poésie. Que ce même matériau se retrouve dans les deux types de textes n'a rien d'étonnant si l'on se souvient que l'émotion est une préoccupation romantique majeure et figure au cœur de l'esthétique keatsienne : la rencontre avec l'objet de beauté, si fugitive soit-elle, donne son élan à la voix poétique ${ }^{2}$.

4 Keats a écrit régulièrement à sa fiancée Fanny Brawne ; quelques poèmes lui sont adressés, ouvertement ou non. Quelques autres encore sont très probablement inspirés non seulement de la relation à Fanny, mais aussi de la relation épistolaire, et du discours épistolaire. Ce discours est construit au fil des lettres par une rhétorique de la lettre d'amour, mélange de code épistolaire et de discours amoureux personnel. Dans la lettre, l'émotion amoureuse est donc l'objet d'une première mise en mots qui précède une nouvelle transformation dans le poème. Partant de cette double transposition, je vais tenter d'examiner comment l'écriture épistolaire est affectée par l'émotion puis comment cette dernière devient matériau poétique. Trois enjeux majeurs s'imposent en effet à l'épistolier et au poète: maîtriser l'émotion, puis écrire le désir et enfin exprimer la souffrance.

\section{Le sentiment amoureux à l'épreuve de l'écriture : entre maîtrise et effusion}

\section{Le masque épistolaire de la maîtrise}

Une des premières lettres à Fanny commence comme une lettre galante dans laquelle l'amoureux déclare son amour et décrit le sentiment qui l'anime. Keats semble suivre la règle épistolaire selon laquelle l'amoureux doit s'exprimer avec retenue et ainsi prouver que la raison n'est pas absente de son sentiment. Parmi les lettres à Fanny, celle-ci est unique en son genre. En effet, elle montre un épistolier qui cherche à construire sa lettre et à emprisonner l'émotion dans les rets du discours :

My dearest Lady,

I am glad I had not an opportunity of sending off a Letter which I wrote you on Tuesday night -'twas too much like one out of Ro[u]sseau's Heloise. I am more reasonable this morning. The morning is the only proper time for me to write to a beautiful Girl whom I love so much: for at night, when the lonely day has closed, and the lonely, silent, unmusical Chamber is waiting to receive me as into a Sepulchre, then believe me my passion gets entirely the sway; then I would not have you see those Rapsodies [sic] which I once thought impossible I should ever give way to and which I have often laughed at in another for fear that you should [think me] either too unhappy or perhaps a little mad. (vol. II, 122)

6 La phrase introductive donne le ton de la lettre, qui ne sera ni emportée ni passionnée. Se déploie ensuite une longue phrase dont la syntaxe complexe parvient à ôter tout effet de spontanéité et contraste avec les syntaxes minimales typiques de l'écriture keatsienne. Les propositions se déroulent et s'imbriquent sur un rythme cadencé avec soin et réglé par des mots charnières comme "then » et "for fear ». Keats cherche le mot juste et entend présenter ses idées avec précision, comme le suggère la suite d'adjectifs «lonely, silent, unmusical Chamber ». Puis les prépositions rejetées en fin d'énoncé sont le signe d'une construction syntaxique non linéaire mais qui repose sur la préconstruction et dont la progression est scrupuleusement contrôlée. D'ailleurs, la lettre est beaucoup plus 
structurée que les autres lettres à Fanny. L'emploi du tiret, si courant dans la correspondance keatsienne, s'estompe au profit d'une ponctuation normale. De plus, la lettre se développe autour de quelques mots qui servent de pivots dans l'enchaînement des idées et donnent au texte une armature certes souple mais bien présente.

7 La lettre comporte par ailleurs son lot d'hyperboles et de compliments («For myself I know not how to express my devotion to so fair a form: I want a brighter word than bright, a fairer word than fair »), comme dans toute lettre. Mais Fanny doit se contenter de déclarations modérées. Celles-ci apparaissent au détour d'une phrase et semblent noyées dans un discours qui cherche avant tout à se distinguer de l'état de folie qu'induit la passion. Au début de la lettre, le complément "a girl whom I love so much », est littéralement incrusté dans la phrase de sorte que la force de la déclaration est atténuée. La passion est ainsi contenue dans le carcan de la phrase, et reléguée à l'arrière-plan de la subordonnée relative. Keats se refuse ici à reproduire l'émotion amoureuse ressentie à vif. L'épistolier présente une lettre à l'écriture visiblement travaillée et mise en ordre afin de contenir l'élan spontané qu'il redoute de montrer.

\section{Désordre amoureux et confusion épistolaire}

8 Au contraire, dans la lettre à Fanny du 16 août 1818, l'émotion atteint totalement l'écriture et se manifeste par une grande confusion de l'expression. Keats ouvre d'ailleurs la lettre en prévenant Fanny: «I see you through a mist ». Ici la lettre est avant tout le lieu de la confusion des sentiments et du discours :

I must remain some days in a mist - I see you through a mist: as I dare say you do me by this time - Believe in the first Letters I wrote you: I assure you I felt as I wrote - I could not write so now - The thousand images I have had pass through my brain - my uneasy spirits - my unguess'd fate - all spread as a veil between you and me - Remember I have had no idle leisure to brood over you - 'tis well perhaps I have not - [...] I am in complete cue - in a fever; and shall in these four Months do an immense deal - This Page as my eye skims over it I see is excessively unloverlike and ungallant - I cannot help it - I am no officer in yawning quarters; no Parsonromeo - My Mind is heap'd to the full; stuff'd like a cricket ball - if I strive to fill it more it would burst - [...]. (vol II, 140-141)

L'écriture prend une allure caractéristique : les phrases sont courtes et entrecoupées de tirets, le rythme est haché, d'autant que le tiret apparaît aussi dans la phrase pour en rompre l'équilibre. L'abondance des tirets, qui remplacent pratiquement tout signe de ponctuation, est typique de l'écriture épistolaire de Keats, mais sa signification n'est pas toujours la même. Ici, elle peut s'interpréter comme un désir de gommer les liens logiques des propositions entre elles, un refus de hiérarchiser les pauses dans le discours, ou une incapacité à le faire. En outre, le discours procède par accumulation d'idées jamais classées les unes par rapport aux autres. Comme l'esprit de l'épistolier, « heaped to the full », la syntaxe tient plus de l'entassement que de l'enchâssement.

Dans la lettre du 13 octobre 1919, le désordre amoureux est tout aussi flagrant, et le style trahit la même incapacité ou le même refus d'organiser le discours. Lieu d'une émotion trop forte qui se libère, la lettre épouse les méandres d'une pensée qui se laisse porter. Ici aussi les phrases sont courtes et leur structure est simplifiée à l'extrême, les tirets récurrents. Mais la spécificité de cette lettre est qu'elle repose sur diverses formes de répétition qui produisent un discours qui ne progresse pas et s'enferme lui-même. La sensation d'impuissance s'exprime aussi dans la récurrence de « cannot » ou de formules 
équivalentes qui apparaissent pas moins de sept fois au cours de la lettre. Puis à la paralysie de la parole s'associent la sensation d'enfermement et la dissolution du moi dans l'autre : «I cannot exist without you - I am forgetful of everything but seeing you again - my life seems to stop here - I see no further. You have absorb'd me. I have a sensation at the present moment as though I was dissolving - (vol. II, 223). D'ailleurs, plus la lettre avance et plus la parole se paralyse, car les redondances soumettent la lettre à une circularité stérile. L'épistolier s'englue dans ce discours qu'il voulait libérateur, comme s'il était effectivement absorbé par son propre discours sur l'être aimé. Ainsi, à la fin de la lettre, la diction fait entendre la difficulté de porter cette émotion. Keats semble même bégayer, buter sur les mots, pris dans une incapacité à se défaire des mots: dépourvues de liens logiques, les phrases s'imbriquent et s'enchaînent au sens propre du mot : «I have been astonished that Men could die Martyrs for religion - I have shudder'd at it - I shudder no more. I could be martyr'd for Religion - Love is my Religion - I could die for that - I could die for you » (vol. II, 223). Soulignons encore un autre trait qui révèle la difficulté à dompter la passion par les mots. À l'image de la déclaration « My love has made me selfish », la lettre est envahie par le pronom "I », ce qui est une entorse majeure à la politesse épistolaire selon laquelle l'épistolier doit parler de lui tout en s'efforçant d'orienter le propos vers les intérêts de l'autre. Avec ce «I» anaphorique, Keats ne cherche nullement à se mettre à la portée de sa destinataire car la lettre a pour seul objet l'expression d'un sentiment d'emprisonnement. Mais cette manifestation apparemment solipsiste sonne aussi comme une ultime tentative discursive pour faire subsister ce moi qui se dit absorbé par sa bien-aimée.

\section{La transposition dans " To Fanny »}

11 Le poème To Fanny transpose cette écriture épistolaire qui oscille entre la tentative pour ordonner l'émotion et le désir de la reproduire fidèlement dans toute son effusion. To Fanny n'imite pas une lettre en particulier mais s'inspire de certains traits de l'écriture épistolaire, les reconstruit et les ordonne différemment. Voici les deux premières strophes du poème :

To Fanny

Physician Nature! let my spirit blood!

$O$ ease my heart of verse and let he rest:

Throw me upon thy tripod, till the flood

of stifling numbers ebbs from my full breast.

A theme! a theme! Great Nature give a theme:

Let me begin my dream.

I come - I see thee, as thou standest there,

Beckon me out into the wintry air.

Ah! Dearest love, sweet home of all my fears

And hopes, and joys and panting miseries, -

To-night, if I may guess, thy beauty wears

A smile of such delight,

As brilliant and as bright

As when with ravished, aching, vassal eyes,

Lost in a maze,

I gaze, I gaze! [...]. 
D'abord, le locuteur et la destinataire du poème semblent rejouer les rôles attribués à Fanny et à Keats tels qu'ils sont représentés dans les lettres. Les images employées sont similaires : par exemple, au vers 14, l'âme se dit malade d'amour, l'amant est soumis. Or «aching» et «vassal» font partie intégrante du discours amoureux épistolaire. «Physician » est aussi un terme que l'on trouve dans les lettres, même si c'est alors Fanny et non la nature, qui guérit l'amoureux de son mal. De même, tout comme l'épistolier qualifie son amour de ferveur proche de la révérence religieuse («Love is my Religion », vol. II, 223 ; « The power of your benediction [...] is like a sacred Chalice once consecrated and ever consecrate ", vol. II, 270), le poète exploite la métaphore religieuse à la fin du poème :

Let None profane my Holy See of Love,

Or with a rude hand break

The sacramental cake. (vers 51-53)

13 Comme dans plusieurs des lettres, la thématique est l'expression douloureuse de la jalousie, la peur d'être délaissé, mais aussi la possessivité qui apparaît dans les expressions qui désignent la femme aimée : «my silver moon » (vers 18), « my Fanny » (vers 33) et «my feast » (vers 17) répondent aux appellatifs affectueux qui ouvrent les lettres. Mais les possessifs, et « My feast » en particulier, rappellent peut-être en même temps le désir de revanche de l'épistolier qui se disait absorbé par sa bien-aimée. D'ailleurs, et ce malgré la position de victime du locuteur, l'effort pour que le sujet «I » survive apparait de manière cyclique à la fin de presque chaque strophe. Outre les nombreuses occurrences de «I " et de "me » à l'intérieur des strophes, la présence du locuteur revient comme un refrain sous diverses formes. «For me» résonne comme un écho dans tout le texte : le cœur du poème n'est pas celui de la femme aimée, mais bien celui du locuteur qui souffre. Comme l'annonce la première strophe, le poème dédié à Fanny ne chante pas les qualités de celle-ci mais ne sert qu'à libérer le locuteur de son obsession. À l'image de la lettre d'amour qui peine à consolider le lien, le poème pervertit le genre de l'éloge propre à l'ode et la transforme en ode à la souffrance du locuteur. Comme ces lettres qui se transforment en longues plaintes, le poème supplie, s'épanche mais ne loue pas, et comme seule preuve d'amour apporte sa complainte.

Aussi, la diction du poème reproduit celle des lettres où l'épistolier semble avoir des difficultés d'élocution. D'abord, le poème est envahi par les tirets: le poète en use ici beaucoup plus que de coutume, au moins une fois à chaque strophe, ce qui rappelle, dans une moindre mesure, la ponctuation anarchique des lettres. Mais surtout, dans presque chaque strophe, le locuteur trébuche sur un mot dans un même vers et trahit là une impuissance, une incapacité à se servir des mots. Ensuite, de nombreuses répétitions à l'intérieur d'une strophe ou d'une strophe à l'autre produisent un effet similaire et amplifient la force de la supplication: "Physician Nature » (vers 1), «Great Nature! » (vers 5). «[L]et » est récurrent dans tout le poème, et enfin «for me » apparaît aux vers 24,25 et 30. Ainsi le bégaiement, qui dans la lettre est facteur de désordre dans le discours et signe de confusion de l'esprit, devient ici un élément du rythme poétique.

Quel est l'effet de la transposition ? De la forme épistolaire il ne subsiste plus que les interpellations (" dearest love » vers 9, « sweet love », vers 25, « my Fanny », vers 33 et "sweet Fanny", vers 42). Le spectre de la lettre refait surface brièvement dans la reconstitution de la réaction de la destinataire du poème, stratagème couramment employé par Keats pour rendre sa lettre plus vivante : « Why this, you'll say - my Fanny! is not true » (vers 33). Hormis ces quelques détails, la lettre paraît désormais très 
lointaine et, on l'a vu, l'objet du poème n'est plus de s'adresser à Fanny. Aussi la forme du poème est très régulière. La solidité de la forme s'oppose donc totalement à la confusion épistolaire. Le poème procède donc à une mise en forme de l'émotion et du désordre amoureux propre aux lettres. Le moule de l'ode, la contrainte de la rime et celle du rythme viennent contenir le flot saccadé et répétitif typique du propos épistolaire. Dans ce poème qui met en scène le mariage de l'émotion et de la maîtrise, les répétitions qui sont censées imiter l'état de confusion de l'amoureux fondent l'orchestration d'un chant au tempo relativement régulier. Ainsi, la mise en poème est le geste qui rend l'expérience fictionnelle. Comme pour réduire l'impact de la douleur et annihiler son emprise, Keats utilise l'émotion dans un texte régi par la mécanique du mètre et de la rime.

Cette ode est le seul poème adressé explicitement à Fanny et à ce titre bénéficie d'un statut particulier. Il est aussi le poème qui semble le plus fidèlement imiter le discours épistolaire en lui empruntant thèmes, images et diction. Car faire référence explicitement à l'expérience du poète n'est pas anodin. En transposant dans un poème la forme de l'adresse inhérente à la lettre, Keats modifie la représentation du lien amoureux, d'abord parce que le discours intime est rendu public. Le statut du poème pourrait paraître ambigu car il est tout de même adressé à Fanny. Mais, justement, la double adresse, explicite à Fanny, implicite au public, sert peut-être à signifier qu'il n'y a plus de destinataire privilégié et que le poème n'appartient pas à Fanny, alors que les lettres ne s'adressent qu'à elle. Lecteurs du poème, nous ne nous sommes pas voyeurs d'une relation intime, comme nous pouvons l'être en lisant les lettres. Le poète se dépossède alors de son expérience en l'offrant à tous, et l'émotion originelle est transformée en émotion poétique potentiellement universelle, point de départ du poème et aussi point d'arrivée, du côté du lecteur.

17 Ainsi, le passage de l'un à l'autre nous montre très précisément comment le poème lyrique se construit et comment, même s'il utilise l'expérience émotionnelle, il s'en distingue, puisque l'adresse n'est qu'un artifice. Par ailleurs, lettres et poèmes se dissocient sur le rapport au temps. La correspondance est inscrite dans le temps de manière précise, elle est datée, c'est même sa raison d'être. Le poème lyrique se construit en revanche sur une mémoire de l'émotion et il est hors du temps. Transformer le discours amoureux épistolaire en poésie lyrique permet alors la mise à distance de l'émotion par la décontextualisation. En revanche, éloigné de la sphère référentielle, le poème procède à une forme de sacralisation de l'expérience, car l'émotion, matière première du texte, est transposée hors du temps pour devenir une parole qui s'adresse à tous. Pourtant, et c'est là toute l'ambiguïté et toute la richesse du poème lyrique, ce chant qui se veut universel est bien l'œuvre d'un sujet qui s'incarne dans une voix. Par rapport à la lettre, dont le rapport à l'émotion réelle est simple (il s'agit de l'exprimer ou de la dissimuler), le poème effectue un travail complexe: il se construit en utilisant l'expérience de l'émotion tout en la mettant à distance, il nie l'identité stricte avec le sujet biographique tout en s'en rapprochant pour reconstruire un autre sujet ${ }^{3}$. Qu'il lui ressemble et qu'il s'en inspire est indéniable, mais le poème apporte la liberté de tout dire ou de dire plus sans que l'auteur doive assumer ces paroles. Il n'encourt donc pas le risque de braver les conventions. Alors que l'épistolier ne sait que faire de son sentiment et hésite entre effusion et maîtrise de soi, le poème élude ces questions et libère l'écriture d'une contrainte pesante. Il en est de même dans le cas de l'expression du désir, embarrassant pour l'épistolier, inspirateur pour le poète. 


\section{Écrire le désir}

\section{La place du corps dans les lettres} trahissent le désir de l'épistolier. Amoureux sincère et épistolier accompli, Keats ne manque pas de louer la beauté de Fanny et prouve ainsi la force de son sentiment amoureux. Mais pour communiquer à sa correspondante son désir, Keats doit utiliser un langage qui le laisse transparaître sans pour autant briser trop ouvertement les règles de bienséance. D'ailleurs, dans une réponse de Keats à Fanny - il s'agit de la lettre citée précédemment - on comprend qu'elle refuse à son fiancé le droit de parler de sa beauté ${ }^{4}$, y voyant peut-être le risque d'une érotisation inconvenante de son corps. Keats ne respecte pas totalement ce vœu, mais il se garde bien en revanche de détailler ses descriptions. l'épistolier ne cherche pas à souligner la particularité. Il se contente souvent d'une figure abstraite aux contours vagues, évoquée de manière répétitive dans des termes généraux et convenus: "Beautiful» ou "Beauty ", «bright», «fair ", "sweet », « delicate ", « loveliness », « graceful » restent les plus courants.

Si peu de recherche peut étonner de la part d'un poète tel que Keats. Ainsi, l'amoureux des images et des phrases délicates n'utilise ni la métaphore ni même la comparaison pour peindre ce corps qu'il désire tant, mais le plus souvent la qualification adjectivale, ou le substantif "your Beauty». Paradoxalement, la langue de l'éloge ne cherche pas à être elle-même objet de beauté. Ainsi, le lieu commun pour évoquer la beauté, qui effleure sans atteindre le corps désiré, est peut-être la garantie que le discours ne glissera pas vers des propos déplacés. Mais sans doute Keats cherchait-il moins à décrire ce corps - on connaît son peu de goût pour la description - qu'à lui donner une présence érotique par des moyens plus détournés.

21 En effet, le corps de Fanny est érotisé à travers un discours qui sans cesse mentionne les lèvres de Fanny, ce qui permet d'envisager la possibilité du baiser et donc d'érotiser le discours. Ainsi se construit, lettre après lettre, une image tronquée du corps de Fanny qui fonctionne comme une synecdoque à répétition : « Will you confess this in the Letter you must write immediately and do all you can to console me in it - make it rich as a draught of poppies to intoxicate me - write the softest words and kiss them that I may at least touch my lips where yours have been » (vol. II, 123). À travers le mot « lips » convergent toute la sensualité du corps de Fanny et toute l'expression du désir de l'épistolier. Ce dernier se délecte du mot et s'en sert comme du support du fantasme. Tandis qu'ici les mots se font caresses («soft words»), le processus inverse est employé dans une autre lettre où le corps féminin devient une construction verbale dans le souvenir de l'amoureux: "I shall kiss your name and mine where your Lips have been - Lips! Why such a prisoner as I am talk about such things! » (vol. II, 270). Peu à peu, l'emprise du désir sur l'épistolier est telle qu'il soumet le langage à son obsession. Pour désigner la jeune femme, le pronom "you» disparaît au profit de la synecdoque : "Your lips are growing sweet again », écrit-il pour signaler à Fanny que l'image de celle-ci surgit dans son esprit et l'empêche de travailler. 


\section{Érotisme et fantasme de totalité dans les poèmes}

Qu'en est-il dans les poèmes inspirés de la relation à Fanny ? Le sonnet " The day is gone » apporte un élément de réponse puisqu'il prend pour objet le corps féminin, le détaille et l'érotise :

The day is gone and all its sweets are gone!

Sweet voice, sweet lips, soft hand and softer breast,

Warm breath, light whisper, tender semi-tone,

Bright eyes, accomplish'd shape, and lang'rous waist!

Faded the flower and all its budded charms,

Faded the sight of beauty from my eyes,

Faded the shape of beauty from my arms,

Faded the voice, warmth, whiteness, paradise,

Vanish'd unseasonably at shut of eve [...]. morcelé par un œil sélectif. Le locuteur ne voit ni une personne identifiée, ni un corps dans son ensemble, mais seulement des atouts érotiques qui composent un portrait tronqué. Comme dans la lettre, le discours est fondé sur le manque et l'absence qui attisent le désir et revêtent une dimension érotique. Mais celle-ci, convertie en matériau poétique, s'incarne aussi dans le tempo. La régularité du rythme dans le deuxième vers suggère un balancement serein et voluptueux ; "sweet " et "soft ", parce qu'ils sont répétés dans un même vers où ils occupent à chaque fois la même place accentuelle, sont comme neutralisés par rapport à " voice ", « lips ", " hand ", «breast ", eux-mêmes mis en lumière car ils semblent alors doublement accentués. Le rythme lancinant porté par les anaphores «faded » devient obsessionnel à mesure que le poème progresse. Le poème garde sans doute une certaine retenue, car il ne chante pas la fusion des corps, mais la mémoire de l'image et une rencontre assez furtive. Cependant, la voix poétique ne réduit pas le corps à un seul de ses attraits. Elle se démarque donc de l'écriture épistolaire qui cherche à mieux contenir le désir en s'interdisant l'évocation trop explicite du corps féminin.

Dans «I cry your mercy - pity - love
s'exprime avec davantage de fougue

I cry your mercy - pity - love! - aye, love,

Merciful love that tantalizes not,

One-thoughted, never wandering, guileless love,

Unmask'd and being seen - without a blot!

0 , let me have thee whole, - all, -all - be mine!

That shape, that fairness, that sweet minor zest

Of love, your kiss, those hands, those eyes divine,

That warm, white, lucient, million-pleasured breast, -

Yourself -your soul - in pity give me all,

Withhold no atom's atom or I die

Or living on perhaps, your wretched thrall,

Forget, in the mist of idle misery,

Life's purposes, - the palate of my mind

Losing its gust, and my ambition blind.

Comme dans le poème précédent, le texte reprend certains traits de l'écriture épistolaire du désir, comme la tendance à morceler le corps et le choix d'évoquer toujours les mêmes attraits («shape», « kiss», « hands», « eyes", «breast») à travers les qualificatifs que 
l'on retrouve aussi bien dans les lettres que dans le sonnet précédent («sweet», "fairness", "warm», "white»). Plus que d'une paresse de l'imagination, il faut sans doute voir là le signe du retour obsessionnel sur le même et la même. Si la beauté n'a qu'un seul visage, c'est qu'elle n'a qu'un seul modèle, comme un repère immuable autour duquel la voix poétique se déploie en diverses directions. Dans « The day is gone and all its sweets are gone », la sensualité du portrait est révélée par le souvenir, qui prend le temps de savourer chaque mot. Ici la sensualité cède la place au désir de posséder la totalité de l'autre, désir si impérieux qu'il prend quasiment la forme de l'injonction dont la détermination s'incarne dans "thee whole ", «all, - all », qui résonne encore avec effroi dans « thrall ». Ce désir de totalité contraste avec le discours épistolaire dans lequel l'amoureux ne réclame que la possession des lèvres.

Aussi le balancement sensuel du sonnet précédent est oublié et se change en martèlement. Les vers quatre à neuf en particulier semblent énoncés par un locuteur qui hoquette et peine à réguler son débit: le rythme iambique se brise dans les nombreuses ruptures imposées par la ponctuation et dans l'enjambement entre les vers 6 et 7. Aussi «all, - all», puis "yourself - your soul», figurent la voix qui se veut lourdement insistante et persuasive, l'amant qui se fait plus pressant et ne cherche pas à construire un rythme régulier. Aussi, les rôles s'inversent si on les compare aux rapports entre l'épistolier et sa destinataire. Le corps féminin est dans le poème un objet à dévorer («[...] the palate of my mind / Losing its gust [...]»), tandis que dans les lettres il est dévorant. Le discours amoureux sur le corps féminin se situe dans le prolongement du discours épistolaire, mais à la fois se transforme dans la poésie, à travers une transposition qui permet à la voix de s'évader du carcan social et du sentiment amoureux, dans lesquels l'épistolier se dit emprisonné.

\section{« [A] dying kind of lover » (vol. II, 281) : l'émotion transfigurée}

\section{La correspondance moribonde}

Dans sa manière d'affronter et de mettre en mot la souffrance d'aimer, Keats rejoint les représentations de l'imaginaire romantique en associant amour et maladie. Plusieurs des dernières lettres à Fanny, alors que le poète malade prend peu à peu conscience qu'il est condamné, superposent au discours amoureux le discours sur la maladie et prennent globalement deux directions. D'abord on y lit ou on croit y lire une absence d'émotion, dans des lettres plus elliptiques à la langue simple et réduites à des billets de quelques lignes informant Fanny essentiellement de son état de santé. Ensuite, dans d'autres lettres, la rhétorique amoureuse se teinte du ton froidement accusateur de l'épistolier qui se dit à la merci de sa bien-aimée et prisonnier d'un amour qu'on ne lui rend pas. L'émotion amoureuse ne se définit plus que négativement car la souffrance morale en devient la seule manifestation. La complainte, le repli sur soi, l'épanchement remplacent l'éloge ou la déclaration, et s'inscrivent dans un discours de plus en plus noir. En effet, le dialogue amoureux est désormais alourdi par la menace de la mort. Le discours s'atrophie d'un côté, en délaissant l'expression des émotions enthousiastes de l'amoureux naïf, mais s'enrichit de l'autre en ayant recours à des images plus sombres qui entraînent le dialogue amoureux dans une spirale destructrice et autodestructrice non moins porteuse d'une écriture esthétique. 
Ainsi le langage amoureux s'infléchit et accentue une tendance déjà présente dès les premières lettres: non seulement la maladie sert très souvent de métaphore aux tourments que l'amour lui inflige, mais le discours sur la maladie, la conception de l'amour et le discours qui l'accompagne semblent peu à peu fusionner à travers un vocabulaire et des références communes. Keats souffre de la séparation et des aléas de la relation et l'exprime sans cesse par le vocabulaire de la douleur physique. Par exemple, « ache » est récurrent ${ }^{5}$; penser à Fanny coincide avec un moment de fièvre : «I encourage it and strive not to think of you - but when I have succeeded in doing so all day and as far as midnight, you return as soon as this artificial excitement goes off more severely than the fever I am left in - » (vol. II, 133).

\section{"La Belle Dame Sans Merci » : anesthésie et douleur}

Sur l'autre rive, La Belle Dame Sans Merci puise très certainement son inspiration dans l'expérience amoureuse de Keats. Sans doute parce que la mise en scène de la rencontre qui sépare les êtres plutôt que de les rapprocher semble reproduire la situation des dernières lettres à Fanny. Sans doute aussi parce que le rôle du chevalier dans la ballade, victime du pouvoir maléfique de la dame, n'est pas sans rappeler la façon dont Keats évoque sa soumission à sa fiancée. Pour ces raisons, et ce même si le poème n'est ni adressé Fanny, ni ouvertement inspiré de leur relation, on peut parler de transposition de l'expérience et du discours amoureux épistolaire.

Outre les parallélismes entre les événements réels et les personnages, cette ballade transpose la rhétorique amoureuse défaillante, la transforme et la magnifie en un spectacle éminemment esthétique. Le sentiment morbide et le désespoir qui hantent les lettres deviennent dans la ballade un spectacle qui produit un effet d'horreur. Tout d'abord, dans ce poème qui fait le récit d'une rencontre amoureuse, l'émotion amoureuse et ses transports semblent paradoxalement absents. Puis les fonctionnements structurels du poème semblent atteints par cet état d'anesthésie. La langue épurée et directe, les liens syntaxiques et chronologiques réduits à leur plus simple expression vont dans ce sens : les rapports entre les mots et les événements ne sont jamais explicités, comme si ces liens étaient distendus. La récurrence de "and", que l'on retrouve dans chaque strophe, le plus souvent deux fois, contribue au dépouillement de la langue et à l'impression de simplicité qui émane du poème à la première lecture. «And » marque la succession temporelle des événements sans les situer précisément les uns par rapports aux autres : il correspond à l'absence de hiérarchisation des éléments, incarne le refus d'expliciter et permet au discours de rester énigmatique. L'ellipse, comme figure majeure du poème, atteint ainsi la représentation des liens sous toutes leurs formes, qu'il s'agisse du rapport entre les êtres ou du rapport entre les événements.

Ensuite, l'obsession de la mort et la souffrance qui en découle, qui toutes deux enrayent le fonctionnement du discours amoureux dans les dernières lettres, sont transposées dans le poème et prennent la forme d'une paralysie générale. La maladie du corps et de l'âme s'étend au monde extérieur et provoque des contaminations en chaîne. La mort est partout: le chevalier est pâle et souffrant, presque un cadavre en devenir, la nature silencieuse, les plantes desséchées et la colline froide, les princes et les rois que le héros rencontre dans son cauchemar agonisent. La présence de la mort se fait plus concrète et pèse à travers des détails macabres : «I saw their starv'd lips in the gloam / with horrid 
warning gaped wide", (vers 41-42). Alors que dans les lettres à Fanny, le discours épistolaire évoque la mort de façon abstraite, la mort devient ici spectacle visuel et cru.

Par ailleurs, le poème en entier se fige pour offrir un spectacle immobile et ramassé où tout se confond. D'abord, les mouvements lents du chevalier (« loitering», «pacing», « I sojourn here ») évoquent la paralysie du corps. Mais surtout, le texte repose sur un jeu de variations autour de scènes et de mots répétés qui associent divers éléments du texte de manière transversale. Keats exploite autant que possible un vocabulaire limité et ces mots servent plusieurs fois dans des contextes différents. Ainsi, les anaphores de " and there » vers 31 et 32 puis 34 et 35 , le refrain "on the cold hill's side» vers 36 et 44 , les nombreuses répétitions comme celles de " full », «fairy » ou de " dream », la reprise de «wild» puis la redondance «wild wild eyes» créent des impressions de déjà-vu qui permettent de condenser les images.

De plus, certains procédés de répétition posent des parallélismes entre les personnages et brouillent les identités. D'abord, la diction du chevalier imite celle du locuteur: d'un point de vue rythmique et syntaxique, «I met a lady in the meads » (vers 13) répond à "I see a lily on thy brow» (vers 9). Les rois et les princes sont à leur tour des doubles du chevalier, tous étant frappés de la même pâleur maladive. Le sens du texte passe par la répétition, les personnages naissant de duplications ou d'extensions d'un même personnage. Le même et l'autre s'enchevêtrent dans le poème pour se dénouer dans des jeux de miroirs déformants et trompeurs. Car les différents personnages ont des facettes communes sans se confondre totalement. En conséquence, les différentes scènes semblent se chevaucher de sorte que la narration progresse peu mais se fige dans un déroulement à la cadence lente.

$34 \mathrm{Au}$ lieu d'une parole vivante qui met en scène l'émotion, effet que produit l'adresse épistolaire ou poétique, $L a$ Belle Dame Sans Merci fait entendre une voix engourdie et moribonde, dresse un tableau où l'émotion est comme emprisonnée dans la souffrance. Ainsi, la contamination, origine de la maladie, est transformée en procédé littéraire qui structure tout le texte et le pétrifie dans un monde enfermé sur lui-même et son système. La transposition de la lettre vers le poème donne ainsi à la douleur une dimension esthétique en la transformant en spectacle quasi pictural, fruit de l'inertie liée aux topiques de la mort et de la maladie, mais aussi grâce à un ensemble d'images reliées entre elles. Ainsi, le poème semble fait pour subir une nouvelle transposition vers la représentation picturale. Cette ultime transformation a eu lieu, puisque La Belle Dame Sans Merci a beaucoup inspiré les peintres préraphaélites, notamment Waterhouse, Dicksee et Cowper.

35 Ces quelques études ont montré que l'émotion affecte l'écriture épistolaire et que le discours épistolaire de l'émotion est affecté par l'écriture poétique. La lecture simultanée de la correspondance et des poèmes permet de mettre au jour le processus qui mène de l'expérience émotionnelle à l'écriture épistolaire puis au poème, autrement dit, comment l'émotion devient fiction.

36 Ainsi, la comparaison des lettres d'amour et des poèmes d'amour mène à considérer les deux types de textes non comme des productions parallèles mais comme des lieux d'écriture qui se frôlent, se nourrissent l'un l'autre, qui partagent et parfois échangent leurs fonctions libératrices. La lettre est d'ordinaire pour Keats un espace de liberté où il peut se laisser aller à des fantaisies linguistiques très éloignées de l'univers poétique, à l'abri du regard du public. Mais dans le cas de l'expression du sentiment amoureux, la rhétorique de la lettre d'amour et les interdits sociaux qu'elle impose, ainsi que 
l'engagement intime de l'épistolier dans la relation sont des garde-fous trop modérateurs qui ne conviennent plus. La lettre devient le carcan d'où Keats s'échappe par le langage poétique. L'épistolier aux prises avec sa passion, soumis et «absorbé » par sa fiancée renaît dans les poèmes sous le masque du créateur puissant qui façonne sa destinataire en personnage. Avec ces quelques poèmes d'amour, Keats n'agit pas sur la relation comme il peut le faire dans les lettres, mais il s'offre le privilège de la réinventer dans des scénarios fantasmatiques.

Il semble que le trajet de la lettre au poème ne soit pas réversible, car la lettre serait première. Pourtant, énonciation épistolaire et énonciation poétique se superposent souvent dans la correspondance, et Keats aurait pu envoyer à Fanny les poèmes qu'elle a inspirés (comme il l'a fait dans les cas des épîtres à ses amis et à son frère), mais il s'en est abstenu. La raison en est peut-être justement que ces poèmes sont détachés de l'expérience et écrits dans le but de se hisser hors de la situation épistolaire, tandis que les épîtres sont plus proches de celle-ci et sont donc interchangeables.

\section{BIBLIOGRAPHIE}

Collot, Michel. La Matière émotion. Paris : PUF, 1997.

Rabaté, Dominique. Figures du sujet lyrique. Paris : PUF, 1996.

Rollins, Hyder Edward (ed.). The Letters of John Keats. 2 vols. London: CUP, 1958.

Stillinger, Jack (ed.). John Keats: Complete Poems. Cambridge, MA: The Belknap Press, 1982.

\section{NOTES}

1. Les références aux lettres proviennent de l'édition suivante : Hyder Edward Rollins, The Letters of John Keats, London: CUP, 1958. Les références indiquent le volume, puis la page.

2. Michel collot, qui a écrit sur l'émotion dans la poésie, décrit le phénomène en ces termes: l'émotion est « une réponse affective d'un sujet à la rencontre d'un être ou d'une chose du monde extérieur qu'il peut tenter d'intérioriser en créant un autre objet, source d'une émotion analogue, mais nouvelle : le poème. » Michel Collot, La Matière émotion, Paris : PUF, 1997, 2.

3. Sur ces questions, on peut se reporter à l'article de Joëlle de Sermet sur l'adresse lyrique. Elle y définit la spécificité du poème lyrique par rapport à d'autres types de discours dont le rapport au réel est différent : «Le poème lyrique progresse à contre-courant de l'autobiographie. Là où cette dernière évolue du singulier vers l'universel (on se souvient des mots placés au seuil des Confessions par Rousseau: "Moi seul. Je sens mon cœur et je connais les hommes") le lyrisme exprime, quant à lui, la généralité du souvenir dans ce qu'il a néanmoins de plus violemment singularisant. " J. de Sermet in Dominique Rabaté (ed.), Figures du sujet lyrique, Paris : PUF, 1996, 84.

4. « Why may I not speak of your Beauty, since without that I could never have lov'd you - I cannot conceive any beginning of such love as I have for you but Beauty. », vol. II, 127. 
5. " [I]n saying this you make me ache the more to be near you "; " you cannot conceive how I ache to be with you "; "Yesterday and this morning I have been haunted with a sweet vision - I have seen you the whole time in your shepherdess dress. How my senses Have ached at it! » (vol. II, 126, 132, 290).

INDEX

oeuvrecitee La Belle Dame Sans Merci, To Fanny 